\title{
Characterization of Kinase Expression Related to Increased Migration of PC-3M Cells Using Global Comparative Phosphoproteome Analysis
}

\author{
YAN GAO ${ }^{1}$, YUN-SOK HA ${ }^{2}$, TAE GYUN KWON ${ }^{2,3}$, YOUNG-CHANG CHO ${ }^{4}$, \\ SANGKYU LEE ${ }^{1 *}$ and JUN NYUNG LEE ${ }^{2 *}$ \\ ${ }^{1}$ BK21 Plus KNU Multi-Omics Based Creative Drug Research Team, College of Pharmacy, \\ Research Institute of Pharmaceutical Sciences, Kyungpook National University, Daegu, Republic of Korea; \\ ${ }^{2}$ Department of Urology, School of Medicine, Kyungpook National University, Daegu, Republic of Korea; \\ ${ }^{3}$ Joint Institute for Regenerative Medicine, Kyungpook National University, Daegu, Republic of Korea; \\ ${ }^{4}$ College of Pharmacy, Chonnam National University, Gwangju, Republic of Korea
}

\begin{abstract}
Background/Aim: Prostate cancer $(\mathrm{PCa})$ is the second-most commonly occurring cancer among men, worldwide. Although the mechanisms associated with the progression of castration-resistant prostate cancer (CRPC) have been widely studied, the mechanism associated with more distant metastases from the bone remains unknown. This study aimed to characterize potential pathogenic kinases associated with highly metastatic PCa, that may regulate phosphorylation in extensively involved and diverse signaling pathways that are associated with the development of various cancers. Materials and Methods: A mass spectrometry (MS)based comparative phosphoproteome strategy was utilized to identify differentially expressed kinases between the highly aggressive PCa cell-lines PC-3 and PC-3M. Results: Among 2,968 phosphorylation sites in PCa cells, 151 differently expressed phosphoproteins were identified. Seven motifs: -SP, -SxxE-, -PxS-, -PxSP-, -SxxK-, -SPxK-, and -SxxxxxP-were
\end{abstract}

This article is freely accessible online.

*These Authors contributed equally to this study.

Correspondence to: Jun Nyung Lee, MD, Ph.D., Assistant Professor, Department of Urology, School of Medicine, Kyungpook National University, 807 Hoguk-ro, Buk-gu, Daegu 41405, Republic of Korea. Tel: +82 532002675, Fax: +82 532002027, email: ljnlover@gmail.com and Sangkyu Lee, Ph.D., Associate Professor, College of Pharmacy, Research Institute of Pharmaceutical Sciences, Kyungpook National University, 80 Daehak-ro, Buk-gu 41566, Republic of Korea. Tel: +82 539508571, Fax: +82 539508557, e-mail: sangkyu@knu.ac.kr

Key Words: Metastatic prostate cancer, proteomics, phosphorylation, kinases. found to be highly expressed in PC-3M cells. Based on these motifs, the kinases p21-activated kinase (PAK)2, Ste20-like kinase (SLK), mammalian Ste20-like kinase (MST)4, mitogenactivated kinase kinase (MAP2K)2, and A-Raf proto-oncogene serine/threonine kinase (ARAF) were up-regulated in PC-3M cells. Conclusion: PAK2, SLK, MST4, MAP2K2, and ARAF are kinases that are potentially associated with the progression of increased migration in PC-3M cells and may represent molecule regulators or drug targets for highly metastatic PCa therapy.

Prostate cancer (PCa) is the second-most commonly occurring cancer among men, worldwide $(1,2)$. According to the latest report by the American Cancer Society, in 2020, 191,930 new cases of PCa will be diagnosed and 33,330 individuals will die from PCa, in the United States (3). Unlike other types of cancers, PCa grows very slowly and may not cause symptoms for years, until the cancer cells metastasize out of the prostate, developing to advanced $\mathrm{PCa}$ $(4,5)$. This characteristic has limited most cases of PCa being recognized and treated during the early stages, and prostate-specific antigen technology began to be applied to the detection of primary-stage PCa in $1994(6,7)$.

Because PCa is an androgen-dependent disease (8), androgen deprivation therapy is the standard therapy for the initial stages of advanced $\mathrm{PCa}$; however, treatment can eventually result in the development of castration-resistant $\mathrm{PCa}$ (CRPC) (9). Androgen receptor (AR) inhibition remains a key factor in CRPC therapies. Metastatic CRPC (mCRPC) develops when PCa cells spread to other parts of the body, where they can grow and spread, even under medical treatment (10). According to the "seed and soil" theory, proposed by Paget (11), PCa spreads in the bloodstream as "seeds", which settle in specific organs, which represent 
"soil" (12). Although organs, such as the liver, lungs, and brain, are common targets of PCa colonization, $\mathrm{PCa}$ spreads most predominantly to the bone (13). The transforming growth factor (TGF)- $\beta$ signaling pathway, bone morphogenetic protein (BMP), and the receptor activator of nuclear factor $\mathrm{kB}$ (NF-kB) (RANK)/RANK ligand (RANKL)/osteoprotegerin (OPG) system are known to be important factors in the formation of bone metastases (1416). However, the metastasis process to distant organ such as liver or lung metastasis remains largely unknown $(17,18)$.

Protein phosphorylation is the most common posttranslational modification observed in proteins (19, 20). Dynamic protein phosphorylation plays a vital role in the activation and deactivation of enzymes and receptors, which are phosphorylated and dephosphorylated by kinases and phosphatases, respectively (21). Protein kinases represent a large category containing various enzymes that catalyze protein phosphorylation (22). Kinase inhibitors have played increasingly prominent roles in the treatment of cancer and other diseases (23). The phosphoinositol-3 kinase (PI3K)/ protein kinase b (AKT)/NF-kB/BMP-2-Smad (24), phosphatase and tensin homolog (PTEN)/PI3K/AKT (25), and PI3K/AKT/mammalian target of rapamycin (mTOR) signaling pathways regulate tumor cell metastasis and invasion in $\mathrm{PCa}$ (26). However, the roles played by kinases in the progression of highly metastatic PCa remains underexplored.

In this study, we performed a global, comparative phosphorylation analysis on the PCa cell lines PC-3 and PC$3 \mathrm{M}$. The PC-3 cell line was established in 1979, from a bone metastasis of a grade IV prostate cancer (27), whereas PC$3 \mathrm{M}$ cells are a metastasis-derived variant of PC-3 cells, which present an increased metastatic capacity and have been found to metastasize to the liver (28). This study aimed to characterize differences in kinase expression cancer during $\mathrm{PCa}$ that are associated with the development of increased metastatic capacity. This study is distinct from previous investigations, due to the following aspects: (i) this study represents the first study of global phosphorylation in PC-3 and $\mathrm{PC}-3 \mathrm{M}$ cells, using quantitative proteomics approaches, and (ii) this study focused on the relationships between kinase expression and the transformation into increasingly metastatic PCa cells. Our results provide a phosphoproteome database for the future study of $\mathrm{PCa}$ and suggested that increased kinase expression in $\mathrm{PC}-3 \mathrm{M}$ may be used to diagnose $\mathrm{PCa}$ or represent potential drug targets for the prevention of $\mathrm{PCa}$ progression to a highly metastatic form.

\section{Materials and Methods}

Prostate cancer cell culture. The PCa cell lines PC-3 and PC-3M were purchased from Korea Cell Line Bank (Seoul, Republic of Korea). PC-3M cells were cultured in RPMI 1640 medium (K0R0) (Thermo Fisher Scientific, Waltham, MA, USA), containing 10\% fetal bovine serum (HyClone Laboratories, Inc. Logan, UT, USA) and $1 \%$ penicillin-streptomycin (Thermo Fisher Scientific). PC-3 cells were cultured in a stable isotope labeling with amino acids in cell culture (SILAC) RPMI 1640 media containing "medium" (K4R6) lysine and arginine isotopes (Cambridge Isotope Laboratory, Andover, MA, USA). The cell culture incubator was maintained at $5 \% \mathrm{CO}_{2}$ and $37^{\circ} \mathrm{C}$. Cells were subcultures at $70-80 \%$ confluence, using $0.05 \%$ trypsin-ethylenediaminetetraacetic acid (EDTA) (Thermo Fisher Scientific) to detach the cells for subculture.

Protein extraction. PCa cells were washed in ice-cold phosphatebuffered saline (PBS, $137 \mathrm{mM} \mathrm{NaCl}, 2.7 \mathrm{mM} \mathrm{KCl}, 10 \mathrm{mM} \mathrm{Na}_{2} \mathrm{HPO}_{4}$, and $1.8 \mathrm{mM} \mathrm{KH}_{2} \mathrm{PO}_{4}, \mathrm{pH}$ 7.4). After PBS buffer was removed, cells were lysed in ice-cold RIPA buffer (Thermos Fisher Scientific), containing $1 \%$ protease inhibitor and $1 \%$ phosphatase inhibitor (Thermo Fisher Scientific). Cells were lysed by sonication, and the lysates were centrifuged at $16,000 \times \mathrm{g}$, at $4^{\circ} \mathrm{C}$ for $10 \mathrm{~min}$, to remove cellular debris. Clarified lysis supernatants were then transferred to new sample tubes. The protein concentrations were determined using a bicinchoninic acid (BCA) protein assay kit (Thermo Fisher Scientific). Protein lysate was maintained at $-80^{\circ} \mathrm{C}$ until use.

Western blotting analysis. To denature proteins for western blotting analysis, $100 \mu \mathrm{g}$ PCa cell lysate was mixed with $4 \times$ sodium dodecyl sulfate (SDS) sample buffer [200 mM Tris-Cl, pH 6.8, $400 \mathrm{mM}$ dithiothreitol (DTT), $8 \%$ SDS, $0.4 \%$ bromophenol blue, and $40 \%$ glycerol]. Distilled water was used to dilute the mixture to $1 \times$ SDS Protein was denatured by heating at $95^{\circ} \mathrm{C}$, for $5 \mathrm{~min}$. Denatured protein was allowed to cool to room temperature (RT) and then loaded at $10 \mu \mathrm{g}$ per/well in a $10 \%$ SDS-polyacrylamide electrophoresis (SDS-PAGE) gel. The SDS-PAGE gel was run at $120 \mathrm{~V}$, at RT. The protein was then electro-transferred from the gel to a polyvinylidene fluoride (PVDF) membrane (Roche, Basel, Switzerland), by wet transfer. The transfer was performed at a voltage of $90 \mathrm{~V}$, for $120 \mathrm{~min}$, in an ice-cold environment. After transfer, the membrane was blocked with $5 \%(\mathrm{v} / \mathrm{v})$ bovine serum albumin (BSA, GenDEPOT, Barker, TX, USA), in 1× Tris-buffered saline containing Tween-20 (TBST, $137 \mathrm{mM}$ sodium chloride and $20 \mathrm{mM}$ Tris, with $0.1 \%$ Tween-20), at RT for $2 \mathrm{~h}$.

The membrane was then incubated with the following primary antibodies: anti-phosphoserine and anti-phosphohistidine (Abcam, Cambridge, MA, USA) and anti-phosphothreonine and antiphosphotyrosine (Cell Signaling Technology, Beverly, MA, USA). Antibodies were diluted at 1:1,000 (v/v), in 5\% (v/v) BSA, and the membrane was incubated with gentle shaking, at $4^{\circ} \mathrm{C}$ overnight. Membranes were then washed in TBST buffer for $10 \mathrm{~min}, 3$ times, followed by incubation with horseradish peroxidase (HRP)conjugated anti-mouse or anti-rabbit antibody (Cell Signaling Technology, Danvers, MA, USA), at RT for 90 min. The membrane was then washed in TBST for $10 \mathrm{~min}, 3$ times. For visualization, the membranes were dampened using $1 \mathrm{ml}$ ECL reagent (GE Healthcare, Chicago, IL, USA), and exposure was performed using the Image Quant LAS 4000 mini (GE Healthcare, Chicago, IL, USA).

Protein in-solution digestion. Prior to in-solution digestion, protein lysates from SILAC-labeled PC-3 (K4R6) and PC-3M (K0R0) cells were mixed at equal protein amounts. A total of $5 \mathrm{mg}$ mixed protein lysate was utilized for the in-solution digestion (29). Protein lysate was reduced in $5 \mathrm{mM}$ DTT, at $56^{\circ} \mathrm{C}$ for $30 \mathrm{~min}$, and alkylated in 15 $\mathrm{mM}$ iodoacetamide (IAA), at RT for $45 \mathrm{~min}$. The DTT and IAA 
solutions were dissolved in $25 \mathrm{mM}$ ammonium bicarbonate (ABC) buffer. To purify proteins, a 4 -fold lysate volume of $-20^{\circ} \mathrm{C}$ cold acetone was added to the lysate. Protein was precipitated at $-20^{\circ} \mathrm{C}$ for $4 \mathrm{~h}$. The protein lysate-acetone mixture was then centrifuged at $16,000 \times g$, at $4^{\circ} \mathrm{C}$ for $15 \mathrm{~min}$. The supernatant was discarded, and the pellet was re-dissolved in $100 \mathrm{mM} \mathrm{ABC}$ buffer. To perform trypsin digestion, sequencing-grade modified trypsin (Promega Corporation, Madison, WI, USA) was added, at 1:50 (w/w), and incubated at $37^{\circ} \mathrm{C}$, for $12 \mathrm{~h}$. Trypsin digestion was quenched by adding $10 \%(\mathrm{v} / \mathrm{v})$ trifluoroacetic acid (TFA), at a final concentration of $1 \%(\mathrm{v} / \mathrm{v})$ TFA. To remove any undigested proteins, the protein mixture was centrifuged, at $16,000 \times g$, at $4^{\circ} \mathrm{C}$ for $10 \mathrm{~min}$. After centrifugation, the digested peptides were soluble in the supernatant, whereas undigested proteins remained in the pellet. The supernatant was then transferred to a new tube and the peptide concentration was measured by a quantitative, colorimetric peptide assay kit (Thermo Fisher Scientific). The peptide solution was then dried using a speed vacuum, without heating. The dried peptides were maintained at $-80^{\circ} \mathrm{C}$ until use.

Phosphorylated peptides, enriched by the $\mathrm{TiO}_{2}$ column. Phosphorylated peptides were enriched using a $\mathrm{TiO}_{2}$ Phosphopeptide Enrichment Kit (Thermo Fisher Scientific), following the manufacturer's instructions. One milligram of peptides was applied to one $\mathrm{TiO}_{2}$ tip. Eluted peptides were fractionated, using a high-pH, reversed-phase, peptide fractionation kit (Thermo Fisher Scientific) and desalted using styrene-divinylbenzene (SDB) and graphite carbon tips (GL Science, Tokyo, Japan). Eluted, desalted peptides were dried in a speed vacuum, without heating. The dried peptides were maintained at $-80^{\circ} \mathrm{C}$ until use.

LC-MS analysis. Prior to MS analysis, the peptides were desalted using $5 \mu \mathrm{g}$ Zip-tip (Millipore, Milford, MA, USA), according to the manufacturer's instructions. The desalted peptide sample was dissolved in $2.5 \mu$ l solvent A (solvent A consisted of $2 \%$ acetonitrile and $0.1 \%$ formic acid, and solvent B consisted of $98 \%$ acetonitrile and $0.1 \%$ formic acid). The peptide sample was separated using a nano-LC (Eksigent, Dublin, CA, USA), equipped with an autosampler system. A C12 column (Proteo C12, $4 \mu \mathrm{m}$ beads, 90 - $\AA$ pore size, Phenomenex, Torrance, CA, USA) was used to connect the LC with the MS. The C12 column was packed with capillary tubing (length: $10 \mathrm{~cm}$, OD: $75 \mu \mathrm{m}$, ID: $150 \mu \mathrm{m}$, Polymicro Technologies, Molex, Lisle, IL, USA). A 75-min gradient nano-LC was employed to analyze phosphorylated peptides, and the flow rate was set to 300 $\mathrm{nl} / \mathrm{min}$. The procedure began with $6 \%$ solvent B and end with $21 \%$ solvent B. For global proteomics analysis, a $3-23 \%$ solvent B gradient was performed over $60 \mathrm{~min}$, with a flow rate set to 300 $\mathrm{nl} / \mathrm{min}$. For the peptide analysis, the nano-LC was coupled with the LTQ Orbitrap Velos MS (Thermo Fisher Scientific), in positive-ion mode. MS data were acquired in the data-dependent mode. The electrospray voltage was set to $1.8 \mathrm{kV}$. The precursor ion scans, ranging from $\mathrm{m} / \mathrm{z} 300-1,800$, were acquired at a resolution of 60,000 , and the automatic gain control (AGC) target value was $1.0 \times 10^{6}$ for the MS scan. Tandem MS was obtained using the collision-induced dissociation mode. The data-dependent mode results, which produced 20 of the most abundant ions from full scans, were fragmented in high-energy collisional dissociation mode, with $35 \%$ normalized collision energy. The maximum IT was $100 \mathrm{~ms}$ for a full scan and $60 \mathrm{~ms}$ for $\mathrm{MS}^{2}$. The mass data are available via ProteomeXchange, with the identifier PXD016282.
Database search. MS raw files were searched in MaxQuant (version 1.5, http://www.maxquant.org/) against a Homo sapiens database (30). For the global proteomic peptide search, the following parameter settings were used: oxidation and acetyl (protein N-term) were chosen as variable modifications, Trypsin/ $\mathrm{P}$ was chosen as digest enzymes, protein false discovery ratio (FDR) was set at 0.01 , and Lys4 and Arg6 were chosen as the isotope labels. For the phosphorylated peptide analysis data search, phosphorylation (SYTH) was added as an option in the variable modifications. A Homo sapiens proteome database was downloaded from UniProt (https://www.uniprot.org/proteomes/UP000005640) (31). For result filtration, "contamination +" and "reverse +" were removed from the results file, and proteins with "scores" below 40 and phosphorylation "localization prob" below $70 \%$ were filtered from the results.

Bioinformatics analysis. Gene functional annotation and Kyoto Encyclopedia of Genes and Genomes (KEGG) pathway mapping were performed using the database for annotation, visualization and integrated discovery (DAVID, version 6.8, https://david.ncifcrf.gov/), according to a previously published protocol (32). A $-\log _{10}$ value of the $p$-value from Fisher's exact test was used as an enrichment factor, and the top five most enriched GO terms were used in the figure. Gene ontology biological process (GOBP), gene ontology cell component (GOCC), gene ontology molecular function (GOMF), InterPro, and KEGG pathways were analyzed in this study.

To characterize the phosphorylation patterns, motif analysis of the phosphorylated peptides was performed, using Motif-All algorithm (33). The pre-aligned sequences had 15-amino-acid lengths, which were used for the foreground format. Phosphomodified amino acids, including serine, threonine, tyrosine, and histidine, were arranged as the central characters. The $p$-value threshold was set to $10^{-6}$. The sequence $\log$ o was visualized through web plogo (https://plogo.uconn.edu/), after inputting pre-aligned sequences. The background was set to "human", and a sequence logo was generated, according to the detailed methods in a previously published research article (34). Site-specific kinasesubstrate relationships were predicted using the software package iGPS (Version 1.0) (35). Using a previously described analysis procedure, the protein phosphorylation network (PPN) among these activated kinases was visualization using PNC 1.0.

The protein-protein interaction (PPI) network was retrieved from the web resource STRING (Version 10.5 https://string-db.org/) (36). During STRING analysis, the UniProt accession IDs were inputted as the queries, Homo sapiens was chosen as the organism, and the "minimum required interaction score" was set to 0.9 , which indicated the highest confidence. To simplify the display, "disconnected nodes" were hidden from the network.

\section{Results}

Proteomics screening of phosphorylation in PCa cells. To eliminate protein expression level effects on the comparison of phosphorylation abundance, protein expression levels in PCa cells were profiled by SDS-PAGE analysis (Figure 1A), which indicated similar protein expression levels between whole-cell lysates derived from PC-3 and PC-3M cells. Then, the phosphorylation levels in PCa cells were explored by western blotting analysis (Figure 1B). Phosphorylation on 
A

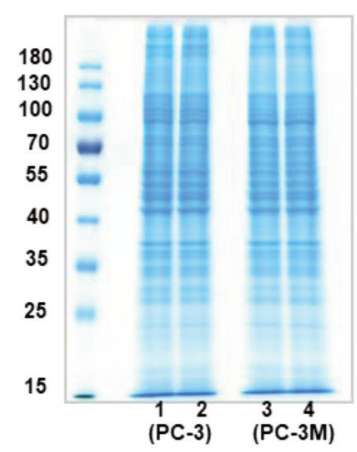

B

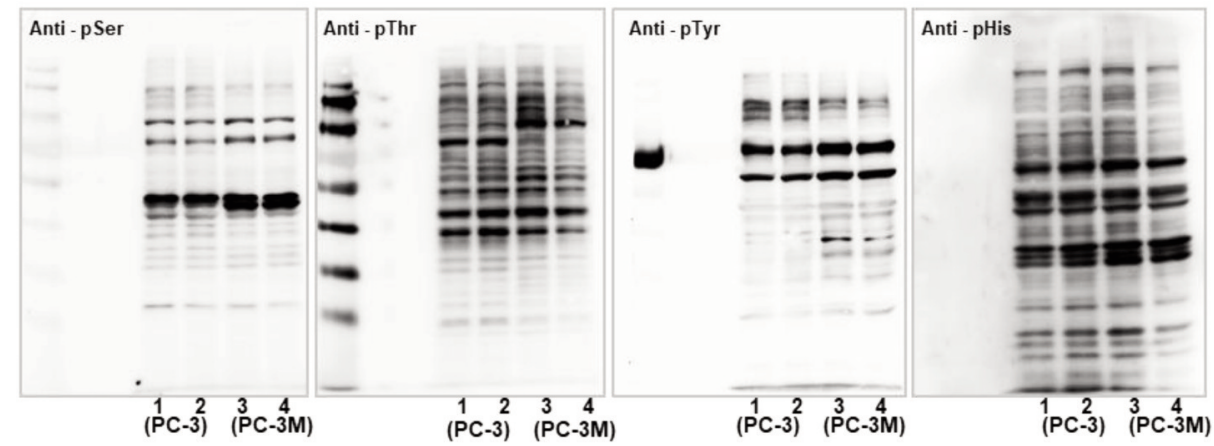

Figure 1. Screening of phosphorylation expression patterns in PCa cells by western blotting analysis. (A) Protein expression profiling in PC-3 and $P C-3 M$ cells by western blotting analysis. Ten micrograms of protein were loaded into lanes 1-4. Lanes 1-2: duplicate loading of protein extracts from PC-3 cells, lanes 3-4: duplication loading from PC-3M cells. (B) Phosphorylation validation in PC-3 and PC-3M cells, by western blotting analysis. Phosphorylation patterns for the amino acids serine (Ser), threonine (Thr), tyrosine (Tyr), and histidine (His) were detected using corresponding antibodies.

the amino acids serine (Ser), threonine (Thr), tyrosine (Tyr), and histidine (His) was detected using relevant antibodies. Western blot results showed different phosphorylation expression levels between $\mathrm{PC}-3$ and $\mathrm{PC}-3 \mathrm{M}$ cells. The expression levels of pSer and pThr in PC-3 cells were relatively higher than in $\mathrm{PC}-3 \mathrm{M}$ cells, whereas pTyr exhibited elevated levels in PC-3 cells for proteins larger than $100 \mathrm{kDa}$, compared to $\mathrm{PC}-3 \mathrm{M}$ cells. Variations in pHis levels between these two PCa cell lines were not obvious.

The proteomics strategy applied to the global profiling of phosphorylation in $\mathrm{PCa}$ cells is depicted in a flow chart (Figure 2A). SILAC PC-3 (K4R6) and PC-3M (K0R0) cells were lysed and mixed at equal protein amounts. After insolution digestion, phosphorylated peptides were enriched by $\mathrm{TiO}_{2}$ bead-embedded tips. Eluted peptides were fractionated by high-pH, reversed-phased chromatography, based on hydrophobicity. The phosphorylated peptides were then chromatographically separated by a nano-LC system and analyzed using an LTQ-Orbitrap Velos. The generated MS spectra were searched using MaxQuant against a Homo sapiens database (version 1.5). A total number of 105,879 MS/MS spectra were generated from the MS raw data. MS raw data are available via ProteomeXchange, with the identifier PXD016282. Data were filtered using a FDR $<1 \%$, score $\geq 40$, and localization prob $\geq 70$. As a result, 2,968 nonredundant phosphorylation sites in 1,316 proteins were identified (Figure 2B). Among them, 2,376 phosphorylation sites in 1,066 phosphoproteins were quantified. Pearson's correlation coefficient analysis was used to evaluate the coefficiency among technical duplicates was shown in (Figure 2C). The R-values reached as high as 0.74 , which indicated that the replicates were highly correlated. Totally, 151 differently expressed phosphoproteins (DEPs) were quantified between PC-3 and PC-3M cells, including 111 upregulated and 50 down-regulated phosphoproteins in $\mathrm{PC}-3 \mathrm{M}$ cells compared with PC-3 cells (under 0.5: down-regulated, over 2: up-regulated).

Functional annotation of differently expressed phosphoproteins. To investigate the functional differences in DEPs between PC3 and PC-3M cells, functional annotation of the DEPs was performed using DAVID (Figure 3). The GOBP results showed that, up-regulated proteins were predominantly enriched in the process of "cell-cell adhesion", followed by "regulation of cellular response to heat", whereas down-regulated proteins were associated with "mRNA splicing, via spliceosome" and "protein sumoylation" processes. The misregulation of cell-cell adhesion in cancer cells has been shown to increase invasion into surrounding stromal tissues, resulting in the formation of advanced cancer (37). GOCC analysis indicated that the DEPs localized to similar cellular components, including the "nucleoplasm" and "cell-cell adherens junctions". In the GOMF analysis, the functions of "poly(A) RNA binding", "cadherin binding involved in cell-cell adhesion", and "protein binding" increased among DEPs. In the InterPro analysis, nucleotide-binding was highly enriched among the DEPs, and "calponin homology domain" and "SIDE domain" were enriched among the up-regulated phosphoproteins, whereas "mini-chromosome maintenance" and "RNA recognition motif domain" were enriched among the down-regulated phosphoproteins. In the KEGG pathway analysis, up-regulated phosphoproteins were primarily associated with "RNA" transport, "Spliceosome", and "ribosome biogenesis in eukaryotes". These functional annotation results indicated that transcripts associated with the mRNA process were highly activated in highly metastatic PCa cells. 
A

$\begin{array}{llll}\text { Digestion TiO2 affinity } \quad \text { Fractionation } \quad \text { LC-MS/MS } & \text { Database search }\end{array}$

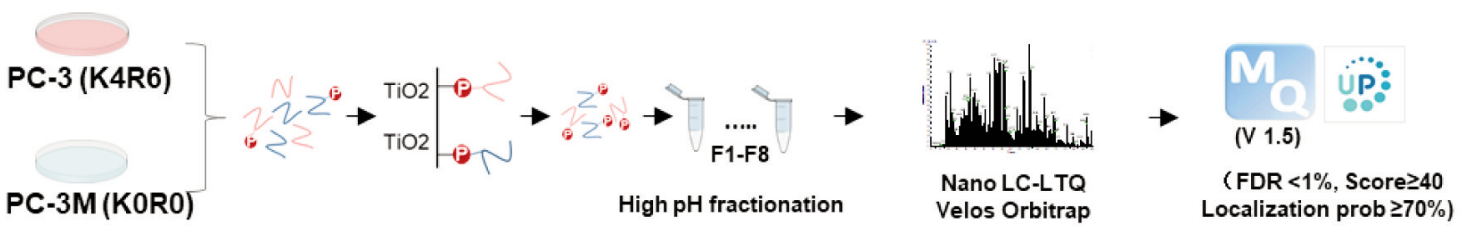

B
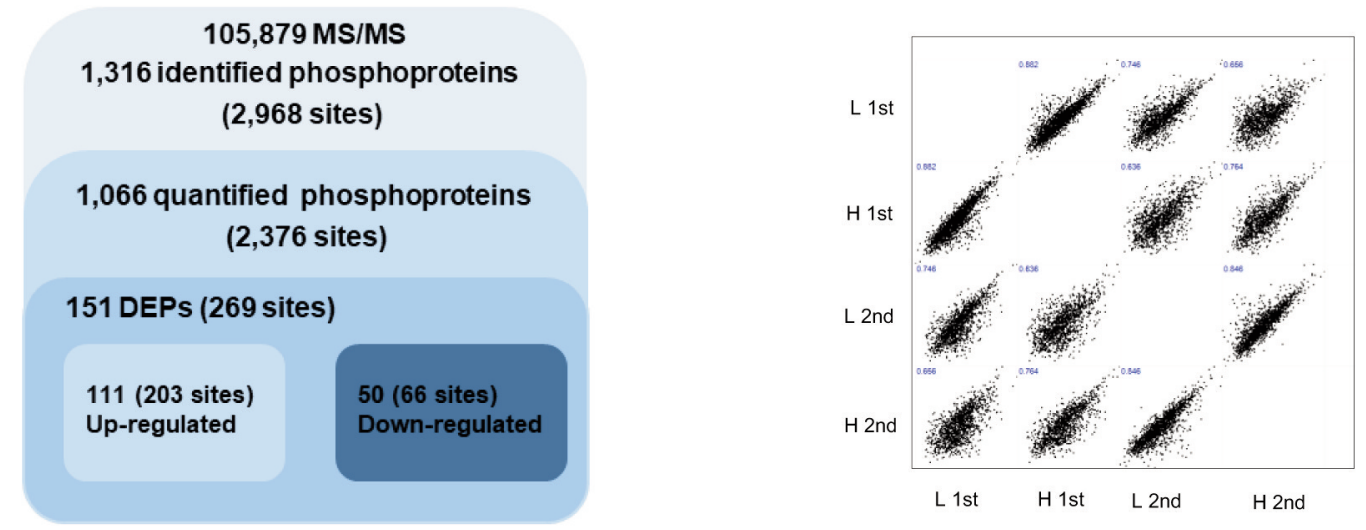

Figure 2. MS-based quantitative proteomic profiling of phosphorylation in PCa. (A) Experimental scheme for quantitative proteomic analysis. PC3 (K4R6) and PC-3M (KORO) were lysed and mixed at equal protein amounts, and tryptic digestion was performed. Phosphorylated peptides were affinity purified using a $\mathrm{TiO}_{2}$ column, and eluted peptides were injected into the nano-LC-MS/MS system. MS spectra were searched in MaxQuant (version 1.5). (B) Overview of the frequency of differentially expressed phosphorylation from proteomics analysis. (C) Correlation of technically replication of MS data.

Phosphorylation motifs in highly metastatic PCa cells. The motifs that surround phosphorylation sites are recognized to be specific substrates for different kinases. To characterize the motifs identified in PC-3 and PC-3M cells, for further kinase prediction analyses, all phosphorylated peptides and DEPs were analyzed using the Motif-All algorithm. The percentage of foreground matches was used as a motif-enrichment index. As a result, 24 motifs, including 18 pSer and 6 pThr motifs, were significantly enriched among our identified phosphosites (Figure 4A). Among the 24 extensively enriched motifs, 8 pSer motifs were differentially expressed between PC-3 and PC-3M cells (Figure 4B). Seven motifs, -SxxE-, -PxS-, -PxSP-, -SxxK-, -SPxK-, -SxxxxxP- and -SP-, were enriched among the up-regulated phosphoproteins in PC-3M cells, whereas the motifs -RxxS- and -SP- were enriched in down-regulated phosphoproteins (The motif -SP- was enriched among both upand down-regulated phosphosites). In addition, -SxxxxxP-was identified as a novel motif, in our study. Motif logos were visualized by the sequence logo tool from plogo (Figure 4B).
Kinases in highly metastatic PCa. To investigate the potential regulatory role played by kinases in the highly metastatic characteristics of PC-3M cell, site-specific kinase and substrate prediction analyses were performed, using the identified DEP phosphosites, through iGPS. As a result, 195 kinases were predicted to regulate the up-regulated phosphosites. Furthermore, a PPN was used to interpret connections between the kinase and specific substrates. A protein network calculator (PNC) was utilized to build the PPNs for the two kinase groups. As a result, 195 kinases (predicted from up-regulated phosphosites) displayed intensive interactions with various substrates (Figure 5A).

To verify our findings and obtain a more reliable result, we co-analyzed the identified kinases using global phosphoproteome and proteome strategies. The co-analysis results revealed that 16 kinases predicted to interact with the identified phosphosites originating from our global proteome analysis (Figure 5B). The results suggested that the CMGC/CDK and AGC kinase groups extensively interacted 


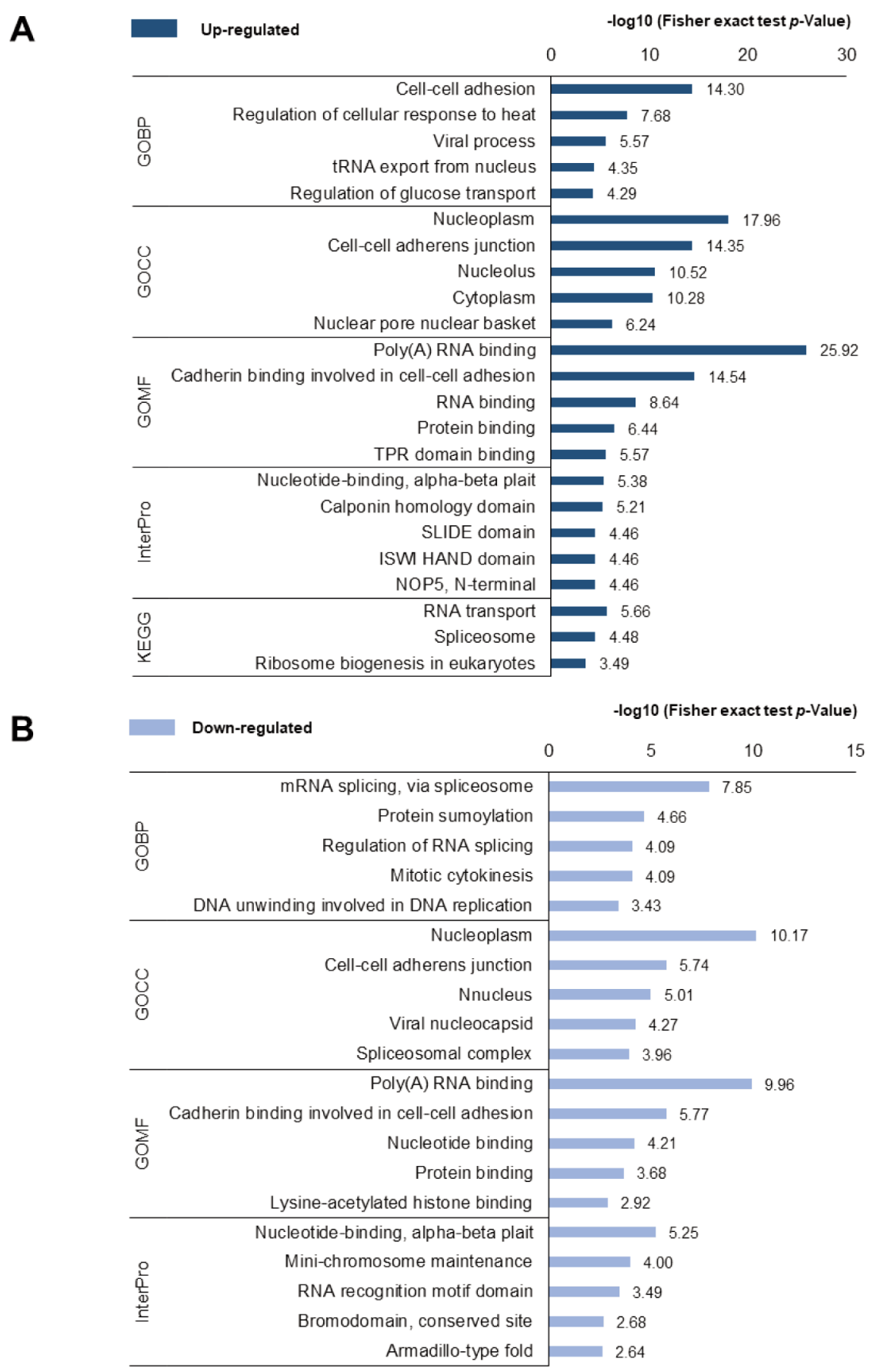

Figure 3. DAVID-generated GO enrichment and KEGG pathway analysis of DEPs. (A) Up-regulated phosphoproteins and (B) down-regulated phosphoproteins. The -log of Fisher's exact test was used to represent the enrichment index. GOBP: Gene Ontology Biological Process, CC: cellular component, MF: molecular function, KEGG: Kyoto Encyclopedia of Genes and Genomes.

with the DEPs. Five kinases, p21-activated kinase (PAK)2, Ste20-like kinase (SLK), mammalian Ste20-like kinase (MST)4, mitogen-activated kinase kinase (MAP2K)2, and A-Raf protooncogene serine/threonine kinase (ARAF) were firstly found to be up-regulated during the progression to PC-3M. PPI networks for these overlapping kinases were visualized using STRING (Figure 5C), which demonstrated that these kinases presented extensive interactions with each other. 


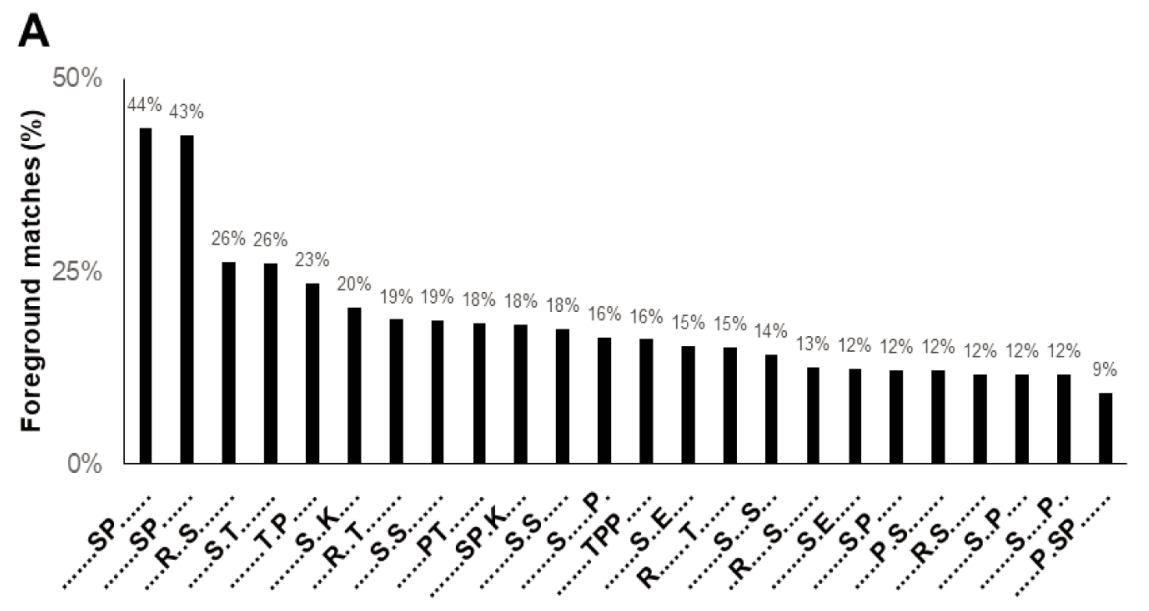

Motifs

B

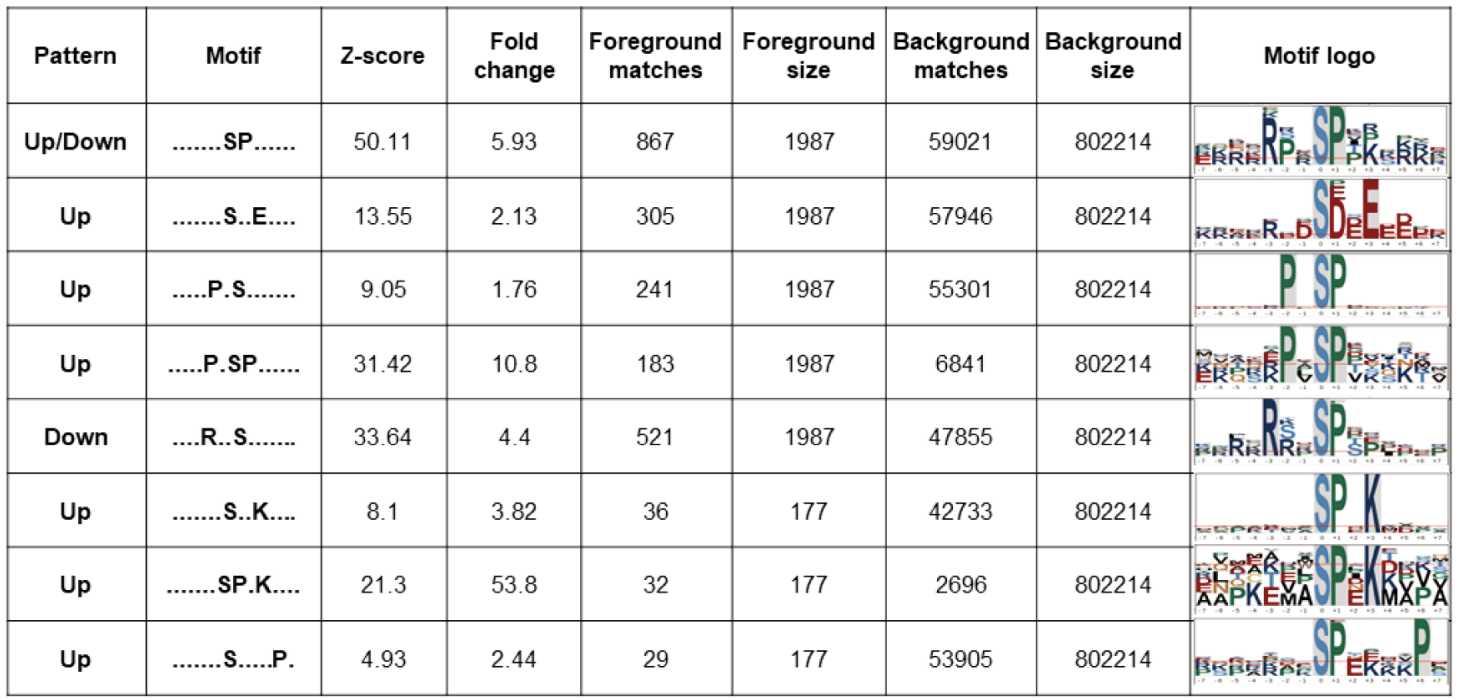

Figure 4. Differentially expressed motifs in PCa. (A) Twenty-four extensively enriched motifs were identified among the phosphosites, including 18 pSer and 6 pThr motifs. Percentage of foreground matches was used as the enrichment index. (B) Differentially expressed motifs between PC-3 and $P C-3 M$ cells. Seven motifs -SxxE-, -PxS-, -PxSP-, -SxxK-, -SPxK-, -SxxxxxP-and-SP-, were up-regulated in PC-3M cells. The motifs -RxxS- and$S P$ - were down-regulated. (Motif-SP-was enriched among both up-and down-regulated phosphosites).

\section{Discussion}

This study integrated two kinds of high-throughput MSbased datasets: global proteome and phosphoproteome. The results demonstrated that several known and novel kinases are associated with the highly metastatic PCa cell line, PC3M (Figure 5B). First, we identified several kinases that are known regulators of $\mathrm{PCa}$. For example, the cyclin-dependent kinases (CDKs), including CDK2, CDK5, CDK6, CDK7, and CDK9, were found to be up-regulated in PC-3M cells. CDKs are known to regulate cell-cycle phases and the overexpression of CDKs can induce cell proliferation (38, 39). A previous study showed that inhibition of CDK2 suppressed $\mathrm{PCa}$ metastases by inactivating PI3K/Akt signaling (40). CDK5, CDK6, CDK7, and CDK9 can suppress CRPC through AR inactivation (41-44). In addition, CDK inhibitors are currently being evaluated in clinical trials (45). Second, serine/threonine-protein kinase N1 (PKN1) and PKN2 have been reported to contribute to the motility of PCa cells, and PKN1 overexpression was observed during clinical CRPC progression (46-48). PKN1 and PKN2 have been suggested to be potential PCa treatment targets. Our 
A

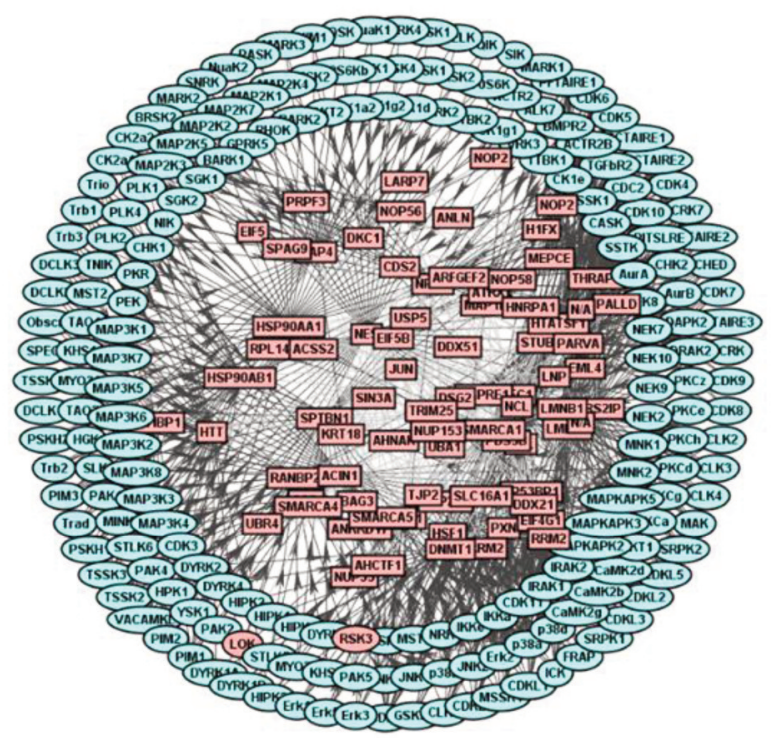

Substrate in Input

Kinase in Input

Regulatory Kinase

B

\begin{tabular}{|l|l|l|}
\hline \multicolumn{3}{|c|}{ Overlaped kinases } \\
\hline Kinas e & Kinase group & $\begin{array}{l}\text { Reference } \\
\text { in PCa }\end{array}$ \\
\hline CDK2 & CMGC/CDK/CDC2 & Known \\
\hline CDK5 & CMGC/CDK & Known \\
\hline CDK6 & CMGC/CDK & Known \\
\hline CDK7 & CMGC/CDK & Known \\
\hline CDK9 & CMGC/CDK & Known \\
\hline PKN1 & A GC & Known \\
\hline PKN2 & AGC & Known \\
\hline PAK2 $*$ & STE/STE20 & Nowel \\
\hline SLK* & STE/STE20 & Novel \\
\hline MST4* & STE/STE20 & Novel \\
\hline MAP2K2 $*$ & STE/STE7 & Novel \\
\hline ARAF* & TKL/RAF & Novel \\
\hline MARK3 & CAMK/CAMKL & Known \\
\hline NEK9 & Other/NEK & Known \\
\hline MAP2K1 & STE/STE7 & Known \\
\hline SRPK1 & CMGC & Known \\
\hline
\end{tabular}

C

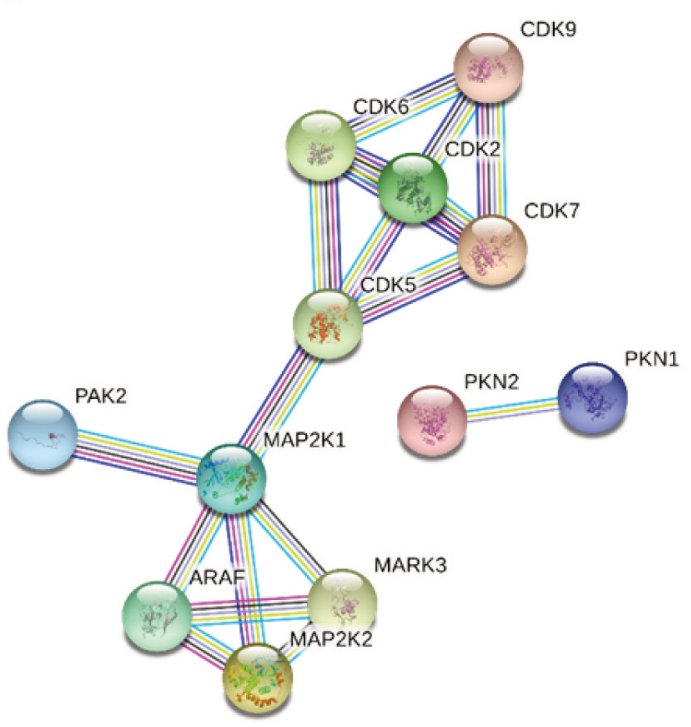

Figure 5. Protein--protein network (PPN) of the upstream kinases in highly metastatic PCa cell. (A) A total of 195 kinase-substrate interactions were predicted from the up-regulated phosphosites. (B) A total of 16 overlapping kinases between phosphosite-predicated and the global proteome experiment were detected. Five kinases $(*)$ are identified as novel kinases regulators during the progression to highly metastatic PCa. (C) PPN of the 16 overlapping kinases. PPN was mapped through the STRING database, with a high confidence value of 0.9.

results are consistent with previous reports, which suggested that the present study's strategy and results are reliable.

In addition, we identified several novel kinase molecules that were associated with the increased migration mechanisms of PC-3M cells. For example, PAK2 was upregulated in PC-3M cells in our study, and the inhibition of PAK2 was able to inhibit the growth of androgenindependent PC3 xenografts (49). SLK is involved in cellcycle progression through the $\mathrm{G}_{2} / \mathrm{M}$ phase $(50,51)$ and is also required for migration in fibroblasts $(52,53)$. SLK is also essential for ErbB2-driven breast cancer cell motility (54). According to these experiments, the elevation of SLK in PC-3M cells suggested that this kinase may increase PCa cell migration through an unknown pathway.

In addition, MST4 is another Ste20 kinase that is known to play a role in PCa progression through signal transduction pathways in the LNCaP, DU 145, and PC-3 cell lines (55). MST4 is not expressed in all benign prostatic hyperplasia 
cases but is expressed in $38.7 \%$ of PCa (56). In our study, MST4 was also found to be up-regulated in PC-3M cells compared to PC-3 cells. These results suggest a positive correlation between MST4 and PCa.

The dual-specificity MAP2K2 can activate both extracellular signal-related kinase (ERK)1 and ERK2 MAPKs $(57,58)$. MAPKs are also known to phosphorylate substrates with the motif -PSP- (59). The increased expression of the motif -PSP- was observed in PC-3M cells (Figure 4B), which indirectly indicated that MAPKs might be activated by MAP2K2. Therefore, MAP2K2 is expected to represent a candidate regulator for cancer cell migration. In addition, ARAF has also been reported to promote the MAPK pathway activation and cell migration $(60,61)$.

In this study, we characterized the global phosphorylation pattern in $\mathrm{PC}-3 \mathrm{M}$ cells, that are highly metastatic PCa cells, to explore the secondary metastatic regulation of $\mathrm{PCa}$ from bone to more distant organs, such as the liver and lung. We identified 2,968 phosphorylation sites and 151 DEPs, and bioinformatics analysis was conducted. We identified 5 novel kinases, PAK2, SLK, MST4, MAP2K2, and ARAF, that are up-regulated in PC$3 \mathrm{M}$ compared to $\mathrm{PC}-3$ cells, which may represent candidates for therapeutic targets against the secondary metastasis of PCa. The present study was limited by a low abundance of identified phosphoproteins, which may result in some potential kinase regulators being overlooked. For example, it was known that tyrosine phosphorylation acts as a central regulator in cell signaling. Relative abundance of pSer:pThr:pTyr (1800:200:1) has been estimated in vertebrate cells. Therefore, characterization of tyrosine phosphorylation is a more challenging task. More recently, this deficiency could be improved by using multiple phosphorylation enrichment kits, specific antibodies affinity beads, or increasing the length of the LC column to improve the peptide sequence coverage.

Taken together, the current analysis indicated that the upregulated kinase: PAK2, SLK, MST4, MAP2K2, and ARAF are concerns to the activation of PC-3M metastasis pathway. Although the current study was investigated in the proteomics field, our result could provide kinase information relating to the prostate cancerization study.

\section{Conflicts of Interest}

The Authors declare no conflicts of interest.

\section{Authors' Contributions}

Conceptualization: Gao, Y., Lee, S. and Lee, J.N.; Methodology and analysis: Gao, Y. and Lee, S.; Resources: Ha, Y., Kwon, T.G. and Lee, J. N.; Data Curation: Gao, Y. Cho, Y. and Lee, S.; Writing: Gao, Y., Lee, S. and Lee, J.N.; Funding Acquisition: Ha, Y., Kwon, T.G., Lee, S and Lee, J.N.

\section{Acknowledgements}

This research was supported by the Basic Science Research Program through the National Research Foundation of Korea and funded by the Korean government (2016R1C1B1011180, 2018R1D1A1A02043591, 2018R1C1B5040264, 2019R1H1A1079839).

\section{Availability of Data and Materials}

The mass spectrometry raw data are available via ProteomeXchange with identifier PXD016282.

\section{References}

1 Ferlay J, Steliarova-Foucher E, Lortet-Tieulent J, Rosso S, Coebergh JW, Comber H, Forman D and Bray F: Cancer incidence and mortality patterns in europe: Estimates for 40 countries in 2012. Eur J Cancer 49(6): 1374-1403, 2013. PMID: 23485231. DOI: $10.1016 /$ j.ejca.2012.12.027

2 Center MM, Jemal A, Lortet-Tieulent J, Ward E, Ferlay J, Brawley $\mathrm{O}$ and Bray F: International variation in prostate cancer incidence and mortality rates. Eur Urol 61(6): 1079-1092, 2012. PMID: 22424666. DOI: 10.1016/j.eururo.2012.02.054

3 Siegel RL, Miller KD and Jemal A: Cancer statistics, 2020. CA Cancer J Clin 70(1): 7-30, 2020. PMID: 31912902. DOI: 10.3322/caac. 21590

4 Teo MY, Rathkopf DE and Kantoff P: Treatment of advanced prostate cancer. Annu Rev Med 70: 479-499, 2019. PMID: 6441973. DOI: 10.1146/annurev-med-051517-011947

5 Stockle M, Junker K and Fornara P: Low-risk prostate cancer prior to or after kidney transplantation. Eur Urol Focus 4(2): 148152, 2018. PMID: 30005996. DOI: 10.1016/j.euf.2018.07.003

6 Pezaro C, Woo HH and Davis ID: Prostate cancer: measuring psa. Intern Med J 44(5): 433-440, 2014. PMID: 24816306. DOI: 10.1111/imj.12407

7 Saini S: Psa and beyond: alternative prostate cancer biomarkers. Cell Oncol (Dordr) 39(2): 97-106, 2016. PMID: 4821699. DOI: 10.1007/s13402-016-0268-6

8 Huggins C and Hodges CV: Studies on prostatic cancer. I. The effect of castration, of estrogen and androgen injection on serum phosphatases in metastatic carcinoma of the prostate. CA Cancer J Clin 22(4): 232-240, 1972. PMID: 4625049. DOI: 10.3322/canjclin.22.4.232

9 Hoang DT, Iczkowski KA, Kilari D, See W and Nevalainen MT: Androgen receptor-dependent and -independent mechanisms driving prostate cancer progression: Opportunities for therapeutic targeting from multiple angles. Oncotarget $8(2)$ : 3724-3745, 2017. PMID: 5356914. DOI: 10.18632 /oncotarget.12554

10 Vlaeminck-Guillem V: When prostate cancer circulates in the bloodstream. Diagnostics (Basel) 5(4): 428-474, 2015. PMID: 4728468. DOI: $10.3390 /$ diagnostics5040428

11 Paget S: The distribution of secondary growths in cancer of the breast. 1889. Cancer Metastasis Rev 8(2): 98-101, 1989. PMID: 2673568. DOI: 10.1016/S0140-6736(00)49915-0

12 Wong SK, Mohamad NV, Giaze TR, Chin KY, Mohamed N and Ima-Nirwana S: Prostate cancer and bone metastases: The underlying mechanisms. Int J Mol Sci 20(10), 2019. PMID: 6567184. DOI: $10.3390 /$ ijms20102587 
13 de Sousa M: Microenvironment to a lymphoid cell is nothing more than interaction with its neighbours. Adv Exp Med Biol 66: 165-169, 1976. PMID: 1083629. DOI: 10.1007/978-1-46134355-4_25

14 Cao Z and Kyprianou N: Mechanisms navigating the tgf-beta pathway in prostate cancer. Asian J Urol 2(1): 11-18, 2015. PMID: 5645057. DOI: 10.1016/j.ajur.2015.04.011

$15 \mathrm{Lu} \mathrm{X}$, Jin EJ, Cheng X, Feng S, Shang X, Deng P, Jiang S, Chang Q, Rahmy S, Chaudhary S, Lu X, Zhao R, Wang YA and DePinho RA: Opposing roles of tgfbeta and bmp signaling in prostate cancer development. Genes Dev 31(23-24): 2337-2342, 2017. PMID: 5795781. DOI: 10.1101/gad.307116.117

16 Christoph F, Konig F, Lebentrau S, Jandrig B, Krause H, Strenziok R and Schostak M: Rank1/rank/opg cytokine receptor system: Mrna expression pattern in bph, primary and metastatic prostate cancer disease. World J Urol 36(2): 187-192, 2018. PMID: 29204705. DOI: 10.1007/s00345-017-2145-y

17 Cotogno PM, Ranasinghe LK, Ledet EM, Lewis BE and Sartor $\mathrm{O}$ : Laboratory-based biomarkers and liver metastases in metastatic castration-resistant prostate cancer. Oncologist 23(7): 791-797, 2018. PMID: 6058331. DOI: 10.1634/theoncologist.2017-0564

18 Zang L, Ma M, Hu J, Qiu H, Huang B and Chu T: The effects of lung and prostate cancer bone metastasis on serum osteoprotegerin levels: A meta-analysis. Sci Rep 5: 18324, 2015. PMID: 4680868. DOI: 10.1038/srep18324

19 Li X, Wilmanns M, Thornton J and Kohn M: Elucidating human phosphatase-substrate networks. Sci Signal 6(275): rs10, 2013. PMID: 23674824. DOI: 10.1126/scisignal.2003203

20 Sacco F, Perfetto L, Castagnoli L and Cesareni G: The human phosphatase interactome: An intricate family portrait. FEBS Lett 586(17): 2732-2739, 2012. PMID: 3437441. DOI: 10.1016/ j.febslet.2012.05.008

21 Ardito F, Giuliani M, Perrone D, Troiano G and Lo Muzio L: The crucial role of protein phosphorylation in cell signaling and its use as targeted therapy (review). Int J Mol Med 40(2): 271280, 2017. PMID: 5500920. DOI: 10.3892/ijmm.2017.3036

22 Cicenas J, Zalyte E, Bairoch A and Gaudet P: Kinases and cancer. Cancers (Basel) 10(3), 2018. PMID: 5876638. DOI: $10.3390 /$ cancers 10030063

23 Gross S, Rahal R, Stransky N, Lengauer C and Hoeflich KP: Targeting cancer with kinase inhibitors. J Clin Invest 125(5): 1780-1789, 2015. PMID: 4463189. DOI: 10.1172/JCI76094

24 Graham TR, Odero-Marah VA, Chung LW, Agrawal KC, Davis $\mathrm{R}$ and Abdel-Mageed AB: Pi3k/akt-dependent transcriptional regulation and activation of bmp-2-smad signaling by nf-kappab in metastatic prostate cancer cells. Prostate 69(2): 168-180, 2009. PMID: 3092122. DOI: 10.1002/pros.20870

25 Mediwala SN, Sun H, Szafran AT, Hartig SM, Sonpavde G, Hayes TG, Thiagarajan P, Mancini MA and Marcelli M: The activity of the androgen receptor variant ar-v7 is regulated by foxo1 in a pten-pi3k-akt-dependent way. Prostate 73(3): 267277, 2013. PMID: 3961010. DOI: 10.1002/pros.22566

26 Chen H, Zhou L, Wu X, Li R, Wen J, Sha J and Wen X: The pi3k/akt pathway in the pathogenesis of prostate cancer. Front Biosci (Landmark Ed) 21: 1084-1091, 2016. PMID: 27100493. DOI: $10.2741 / 4443$

27 Kaighn ME, Narayan KS, Ohnuki Y, Lechner JF and Jones LW: Establishment and characterization of a human prostatic carcinoma cell line (pc-3). Invest Urol 17(1): 16-23, 1979. PMID: 447482.
28 Pettaway CA, Pathak S, Greene G, Ramirez E, Wilson MR, Killion JJ and Fidler IJ: Selection of highly metastatic variants of different human prostatic carcinomas using orthotopic implantation in nude mice. Clin Cancer Res 2(9): 1627-1636, 1996. PMID: 9816342.

29 Kim SC, Chen Y, Mirza S, Xu Y, Lee J, Liu P and Zhao Y: A clean, more efficient method for in-solution digestion of protein mixtures without detergent or urea. J Proteome Res 5(12): 34463452, 2006. PMID: 17137347. DOI: 10.1021/pr0603396

30 Tyanova $\mathrm{S}$, Temu $\mathrm{T}$ and Cox $\mathrm{J}$ : The maxquant computational platform for mass spectrometry-based shotgun proteomics. Nat Protoc 11(12): 2301-2319, 2016. PMID: 27809316. DOI: 10.1038/nprot.2016.136

31 Pundir S, Martin MJ, O'Donovan C and UniProt C: Uniprot tools. Curr Protoc Bioinformatics 53: 1.29.1-1.29.15, 2016. PMID: 4941944. DOI: 10.1002/0471250953.bi0129s53

32 Huang da W, Sherman BT and Lempicki RA: Systematic and integrative analysis of large gene lists using david bioinformatics resources. Nat Protoc 4(1): 44-57, 2009. PMID: 19131956. DOI: 10.1038/nprot.2008.211

33 He Z, Yang C, Guo G, Li N and Yu W: Motif-all: Discovering all phosphorylation motifs. BMC Bioinformatics 12(Suppl 1): S22, 2011. PMID: 3044277. DOI: 10.1186/1471-2105-12-S1S22

34 O'Shea JP, Chou MF, Quader SA, Ryan JK, Church GM and Schwartz D: Plogo: A probabilistic approach to visualizing sequence motifs. Nat Methods 10(12): 1211-1212, 2013. PMID: 24097270. DOI: 10.1038/nmeth.2646

35 Song C, Ye M, Liu Z, Cheng H, Jiang X, Han G, Songyang Z, Tan Y, Wang H, Ren J, Xue Y and Zou H: Systematic analysis of protein phosphorylation networks from phosphoproteomic data. Mol Cell Proteomics 11(10): 1070-1083, 2012. PMID: 3494146. DOI: $10.1074 / \mathrm{mcp} . M 111.012625$

36 Szklarczyk D, Franceschini A, Wyder S, Forslund K, Heller D, Huerta-Cepas J, Simonovic M, Roth A, Santos A, Tsafou KP, Kuhn M, Bork P, Jensen LJ and von Mering C: String v10: Protein-protein interaction networks, integrated over the tree of life. Nucleic Acids Res 43(Database issue): D447-452, 2015. PMID: 4383874. DOI: 10.1093/nar/gku1003

37 Vasioukhin V: Adherens junctions and cancer. Subcell Biochem 60: 379-414, 2012. PMID: 22674080. DOI: 10.1007/978-94007-4186-7_16

38 Sanchez I and Dynlacht BD: New insights into cyclins, cdks, and cell cycle control. Semin Cell Dev Biol 16(3): 311-321, 2005. PMID: 15840440. DOI: 10.1016/j.semcdb.2005.02.007

39 Malumbres M and Barbacid M: Cell cycle, cdks and cancer: A changing paradigm. Nat Rev Cancer 9(3): 153-166, 2009. PMID: 19238148. DOI: 10.1038/nrc2602

40 Yin X, Yu J, Zhou Y, Wang C, Jiao Z, Qian Z, Sun H and Chen B: Identification of cdk2 as a novel target in treatment of prostate cancer. Future Oncol 14(8): 709-718, 2018. PMID: 29323532. DOI: $10.2217 /$ fon-2017-0561

41 Maddox AM, Freireich EJ, Keating MJ, Frasier-Scott KF and Haddox MK: Alterations in human circulating and bone marrow mononuclear cell polyamine levels in hematologic malignancies as a consequence of difluoromethylornithine administration. Invest New Drugs 6(2): 125-134, 1988. PMID: 3139580. DOI: $10.1007 /$ bf00195371

42 Lim JT, Mansukhani M and Weinstein IB: Cyclin-dependent kinase 6 associates with the androgen receptor and enhances its 
transcriptional activity in prostate cancer cells. Proc Natl Acad Sci USA 102(14): 5156-5161, 2005. PMID: 556011. DOI: 10.1073/pnas.0501203102

43 Rasool RU, Natesan R, Deng Q, Aras S, Lal P, Sander Effron S, Mitchell-Velasquez E, Posimo JM, Carskadon S, Baca SC, Pomerantz MM, Siddiqui J, Schwartz LE, Lee DJ, Palanisamy N, Narla G, Den RB, Freedman ML, Brady DC and Asangani IA: Cdk7 inhibition suppresses castration-resistant prostate cancer through med1 inactivation. Cancer Discov 9(11): 1538-1555, 2019. PMID: 31466944. DOI: 10.1158/2159-8290.CD-19-0189

44 Rahaman MH, Kumarasiri M, Mekonnen LB, Yu M, Diab S, Albrecht H, Milne RW and Wang S: Targeting cdk9: A promising therapeutic opportunity in prostate cancer. Endocr Relat Cancer 23(12): T211-T226, 2016. PMID: 27582311. DOI: 10.1530/ERC-16-0299

45 Rizzolio F, Tuccinardi T, Caligiuri I, Lucchetti C and Giordano A: Cdk inhibitors: from the bench to clinical trials. Curr Drug Targets 11(3): 279-290, 2010. PMID: 20210753. DOI: $10.2174 / 138945010790711978$

46 Yang CS, Melhuish TA, Spencer A, Ni L, Hao Y, Jividen K, Harris TE, Snow C, Frierson HF, Jr., Wotton D and Paschal BM: The protein kinase c super-family member $p k n$ is regulated by mtor and influences differentiation during prostate cancer progression. Prostate 77(15): 1452-1467, 2017. PMID: 5669364. DOI: $10.1002 /$ pros. 23400

47 Venkadakrishnan VB, DePriest AD, Kumari S, Senapati D, BenSalem S, Su Y, Mudduluru G, Hu Q, Cortes E, Pop E, Mohler JL, Azabdaftari G, Attwood K, Shah RB, Jamieson C, Dehm SM, Magi-Galluzzi C, Klein E, Sharifi N, Liu S and Heemers $\mathrm{HV}$ : Protein kinase $\mathrm{n} 1$ control of androgen-responsive serum response factor action provides rationale for novel prostate cancer treatment strategy. Oncogene 38(23): 4496-4511, 2019. PMID: 6771259. DOI: 10.1038/s41388-019-0732-7

48 O'Sullivan AG, Mulvaney EP, Hyland PB and Kinsella BT: Protein kinase c-related kinase 1 and 2 play an essential role in thromboxane-mediated neoplastic responses in prostate cancer. Oncotarget 6(28): 26437-26456, 2015. PMID: 4694913. DOI: 10.18632 /oncotarget. 4664

49 Jiang N, Hjorth-Jensen K, Hekmat O, Iglesias-Gato D, Kruse T, Wang C, Wei W, Ke B, Yan B, Niu Y, Olsen JV and FloresMorales A: In vivo quantitative phosphoproteomic profiling identifies novel regulators of castration-resistant prostate cancer growth. Oncogene 34(21): 2764-2776, 2015. PMID: 25065596. DOI: $10.1038 /$ onc.2014.206

50 O'Reilly PG, Wagner S, Franks DJ, Cailliau K, Browaeys E, Dissous C and Sabourin LA: The ste20-like kinase slk is required for cell cycle progression through g2. J Biol Chem 280(51): 42383-42390, 2005. PMID: 16236704. DOI: 10.1074/ jbc.M510763200

51 Wang K, Hong RL, Lu JB and Wang DL: Ste20-like kinase is upregulated in glioma and induces glioma invasion. Neoplasma 65(2): 185-191, 2018. PMID: 9534578. DOI: 10.4149/neo_2018_ 170318 N193
52 Wagner S, Storbeck CJ, Roovers K, Chaar ZY, Kolodziej P, McKay M and Sabourin LA: Fak/src-family dependent activation of the ste20-like kinase slk is required for microtubule-dependent focal adhesion turnover and cell migration. PLoS One 3(4): e1868, 2008. PMID: 2270904. DOI: 10.1371/journal.pone.0001868

53 Wagner SM and Sabourin LA: A novel role for the ste20 kinase slk in adhesion signaling and cell migration. Cell Adh Migr 3(2): 182-184, 2009. PMID: 2679882. DOI: 10.4161/cam.3.2.7229

54 Roovers K, Wagner S, Storbeck CJ, O'Reilly P, Lo V, Northey JJ, Chmielecki J, Muller WJ, Siegel PM and Sabourin LA: The ste20-like kinase slk is required for erbb2-driven breast cancer cell motility. Oncogene 28(31): 2839-2848, 2009. PMID: 19525980. DOI: $10.1038 /$ onc.2009.146

55 Sung V, Luo W, Qian D, Lee I, Jallal B and Gishizky M: The ste20 kinase mst4 plays a role in prostate cancer progression. Cancer Res 63(12): 3356-3363, 2003. PMID: 12810671.

56 Zhang H, Ma X, Peng S, Nan X and Zhao H: Differential expression of mst 4 , stk 25 and pdcd 10 between benign prostatic hyperplasia and prostate cancer. Int J Clin Exp Pathol 7(11): 8105-8111, 2014. PMID: 4270628.

57 Lavoie H, Sahmi M, Maisonneuve P, Marullo SA, Thevakumaran N, Jin T, Kurinov I, Sicheri F and Therrien M: Mek drives braf activation through allosteric control of ksr proteins. Nature 554(7693): 549-553, 2018. PMID: 6433120. DOI: $10.1038 /$ nature 25478

58 Seger R, Seger D, Reszka AA, Munar ES, Eldar-Finkelman H, Dobrowolska G, Jensen AM, Campbell JS, Fischer EH and Krebs EG: Overexpression of mitogen-activated protein kinase kinase (mapkk) and its mutants in nih $3 \mathrm{t} 3$ cells. Evidence that mapkk involvement in cellular proliferation is regulated by phosphorylation of serine residues in its kinase subdomains vii and viii. J Biol Chem 269(41): 25699-25709, 1994. PMID: 7929275.

59 Wang ZK, Wang J, Liu J, Ying SH, Peng XJ and Feng MG: Proteomic and phosphoproteomic insights into a signaling hub role for cdc14 in asexual development and multiple stress responses in beauveria bassiana. PLoS One 11(4): e0153007, 2016. PMID: 4824431. DOI: 10.1371/journal.pone.0153007

60 Wei H, Xu L, Yu M, Zhang L, Wang H, Wei X and Ruan Y: Monocillin ii inhibits human breast cancer growth partially by inhibiting mapk pathways and cdk2 thr160 phosphorylation. Chembiochem 13(3): 465-475, 2012. PMID: 22253097. DOI: $10.1002 /$ cbic 201100558

61 Mooz J, Oberoi-Khanuja TK, Harms GS, Wang W, Jaiswal BS, Seshagiri S, Tikkanen R and Rajalingam K: Dimerization of the kinase araf promotes mapk pathway activation and cell migration. Sci Signal 7(337): ra73, 2014. PMID: 25097033. DOI: $10.1126 /$ scisignal.2005484 